\title{
OPERATORS APPROXIMABLE BY HYPERCYCLIC OPERATORS
}

\author{
JAMES BOLAND
}

\begin{abstract}
We show that operators on a separable infinite dimensional Banach space $X$ of the form $I+S$, where $S$ is an operator with dense generalised kernel, must lie in the norm closure of the hypercyclic operators on $X$, in fact in the closure of the mixing operators.
\end{abstract}

\section{IntRoduction}

Hypercyclic operators $T: X \rightarrow X$ on a Banach space $X$ (those bounded linear operators for which there is a vector $v \in X$ with dense orbit $\left.\left\{v, T v, T^{2} v, \ldots\right\}\right)$ have been the subject of intense study in recent decades and we refer to [3] for a recent overview of the topic. Many problems that were posed about hypercyclicity have now been solved. In particular, it is well known that every separable infinite dimensional Banach space $X$ supports hypercyclic operators and in fact many such operators. We use $\mathcal{B}(X)$ for the bounded linear operators on $X$ (always over $\mathbb{C}$ ) and $\mathcal{H C}(X)$ for the subclass of hypercyclic operators. An elegant result of [4] allows one to conclude that, for $T \in \mathcal{H C}(X)$, the similarity class $\left\{S^{-1} T S: S \in \mathcal{B}(X)\right.$ invertible $\}$ is always dense in the strong operator topology of $\mathcal{B}(X)$ (and consists of hypercyclic operators).

In the operator norm on $\mathcal{B}(X)$, there are some obvious restrictions such as $\|T\|>1$ for $T \in \mathcal{H C}(X)$, but Herrero [6] characterised the norm closure $\overline{\mathcal{H C}(H)}$ of $\mathcal{H C}(H)$ in spectral terms for $H$ a (separable infinite dimensional) Hilbert space. In fact the paper [6] may be seen as flowing from a considerable body of work (see [5, 1]) aimed at characterising the norm closure of the similarity class of a Hilbert space operator, but [6. Remark 2.3] notes that the necessary and sufficient conditions for a Hilbert space operator $T \in \mathcal{B}(H)$ to belong in $\overline{\mathcal{H C}(H)}$ are also necessary for the Banach space case. In the extreme case of pathological Banach spaces $Y$ with few operators, the existence of which was recently established by Argyros and Haydon [2], all operators $T \in \mathcal{B}(Y)$ are of the form $T=\lambda I+K$ with $\lambda$ a scalar and $K$ compact, and

Date: 01:17 Friday $30^{\text {th }}$ July, 2021.

This work was supported by the Science Foundation Ireland under grant 11/RFP/MTH3187. 
then the Herero conditions reduce to $|\lambda|=1$ and $K$ quasinilpotent (spectrum just $\{0\}$ ).

Our main result (Theorem 3.1) implies (Corollary 3.2) that (for $X$ a separable infinite dimensional Banach space) operators $T=\lambda I+N$ with $|\lambda|=1$ and $N \in \mathcal{B}(X)$ nilpotent are all in the operator norm closure of $\mathcal{H C}(X)$. So far we have been unable to decide whether this result remains true with $N$ replaced by a quasinilpotent $K$, but we have some results for special cases of quasinilpotent $K$.

The methods we use to obtain this approximation are largely elementary, based on certain notions of generalised shifts - which may be seen as operators that admit a Jordan form representation in a somewhat loose sense. We rely then on an observation of V. Müller [8, Theorem 1] which is a simplification of [3, Theorem 2.2], attributed to Grivaux and Shkarin, to show that translates of such shifts by the identity operator $I$, or by $\lambda I$ with $|\lambda|=1$, are mixing (and hence hypercyclic).

We recall that $T \in \mathcal{B}(X)$ is called a mixing operator if for each pair $U, V$ of nonempty open subsets of $X$, there is $N$ such that $T^{n}(U) \cap V \neq$ $\emptyset \forall n>N$. We will use $\mathcal{M i x}(X)$ to denote the mixing operators on $X$.

\section{Generalised Shifts}

For $T \in \mathcal{B}(X)$ we use the notation $\mathcal{N}^{\infty}(T)$ for the generalised kernel $\bigcup_{n=1}^{\infty} \operatorname{ker}\left(T^{n}\right)$ and $\mathcal{R}^{\infty}(T)=\bigcap_{n=1}^{\infty} T^{n}(X)$ for the hyperrange.

It will be convenient for us to introduce the (lexicographic) ordering $<$ on $\mathbb{N} \times \mathbb{N}$ given by $(p . q)<(\ell, k)$ if either $p<l$ or $p=\ell$ and $q<k$. Given $(\ell, k) \in \mathbb{N} \times \mathbb{N}$ with $k>1$ we refer to $(\ell, k-1)$ as the immediate predecessor of $(\ell, k)$. This helps to explain the notions of generalised (backward) shift that we will use. Notice that we base these notions of shifts on sets of vectors which span a dense linear subspace, but need not be Schauder bases for $X$.

Definition 2.1. Let $X$ be a separable infinite dimensional Banach space. Given a set of linearly independent vectors $\left\{x_{k}^{\ell}:(\ell, k) \in\right.$ $\{1, \ldots, n\} \times \mathbb{N}\}$ whose span is dense in $X$, we call $S \in \mathcal{B}(X)$ a generalised backward $n$-shift adapted to the set if for each $(\ell, k) \in\{1, \ldots, n\} \times$ $\mathbb{N}, T\left(x_{k}^{\ell}\right)$ is a finite linear combination of $x_{q}^{p}$ with $(p, q)<(\ell, k)$ and is such that the coefficient of the immediate predecessor, $x_{k-1}^{\ell}$, is non-zero when $k>1$. In more formal terms, we insist that

$$
\begin{array}{r}
S x_{k}^{\ell}=\sum_{i=1}^{k-1} a_{i}^{\ell, k} x_{i}^{\ell}+\sum_{i=1}^{\ell-1} \sum_{j=1}^{m_{j}^{\ell, k}} b_{i, j}^{\ell, k} x_{j}^{i} \quad a_{i}^{\ell, k}, b_{i, j}^{\ell, k} \in \mathbb{K}, \\
a_{k-1}^{\ell, k} \neq 0 \text { if } k>1, m_{j}^{\ell, k} \in \mathbb{N} .
\end{array}
$$

Similarly, given a set of linearly independent vectors $\left\{x_{k}^{\ell}:(\ell, k) \in\right.$ $\mathbb{N} \times \mathbb{N}\}$ whose span is dense in $X$, we call $S \in \mathcal{B}(X)$ a generalised 
backward $\infty$-shift (adapted to the set) if for each $(\ell, k) \in \mathbb{N} \times \mathbb{N}, T\left(x_{k}^{\ell}\right)$ is a finite linear combination (2.1).

We think of the sequence $\left(x_{k}^{\ell}\right)_{k=1}^{\infty}$ as the $\ell^{\text {th }}$ chain of the set.

Lemma 2.2. If $S \in \mathcal{B}(X)$ is a generalised $n$-shift or $\infty$-shift adapted to a set $\left\{x_{k}^{\ell}\right\}$, then $x_{k}^{\ell} \in \mathcal{R}^{\infty}(S) \cap \mathcal{N}^{\infty}(S)$ (for each $(\ell, k)$ in the set).

Proof. The proof is a simple induction on $\ell$.

For $\ell=1, S x_{k}^{1}=\sum_{i=1}^{k-1} a_{i}^{1, k} x_{i}^{1} \in \operatorname{span}\left\{x_{i}^{1}: 1 \leq i \leq k-1\right\}, x_{1}^{1} \in \operatorname{ker} S$. Clearly $x_{k}^{1} \in \operatorname{ker} S^{k} \subseteq \mathcal{N}^{\infty}(S)$ for each $k$. Also,

$$
S^{r}\left(\frac{x_{\ell+1}^{1}}{\prod_{i=1}^{r} a_{i}^{1, i+1}}\right)=x_{1}^{1}
$$

and so $x_{1}^{1} \in S^{r}(X)$ for all $r$. Hence $\mathbb{C} x_{1}^{1} \subseteq \mathcal{R}^{\infty}(S)$. Using a subinduction on $k$, assume $x_{i}^{1} \in \mathcal{R}^{\infty}(S)$ for $1 \leq i \leq k$. Then

$$
S^{r}\left(\frac{x_{r+k+1}^{1}}{\prod_{i=1}^{r} a_{i+k}^{1, i+k+1}}\right)=x_{k+1}^{1}+u
$$

with $u \in \operatorname{span}\left\{x_{i}^{1}: 1 \leq i \leq k\right\} \subseteq \mathcal{R}^{\infty}(S)$ and $u \in S^{r}(X) \Rightarrow x_{k+1}^{1} \in$ $S^{r}(X)$ for $r \geq 1$. Hence $x_{k+1}^{1} \in \mathcal{R}^{\infty}(S)$. By induction we get $x_{k}^{1} \in$ $\mathcal{R}^{\infty}(S)$ for all $k$.

Similar arguments (using finiteness of the sums (2.1)) allow us to show the induction step on $\ell$.

The following is a simplified version of [8, Theorem 1] which is a simplification of [3, Theorem 2.2].

Theorem 2.3. Let $X$ be a separable Banach space, $S \in \mathcal{B}(X)$ with $\mathcal{N}^{\infty}(S) \cap \mathcal{R}^{\infty}(S)$ dense in $X$ and let $\lambda \in \mathbb{C}$ with $|\lambda|=1$. Then $\lambda+S \in$ $\mathcal{M i x}(X)$.

Corollary 2.4. If $X$ is a Banach space, $S \in \mathcal{B}(X)$ a generalised $n$ shift or $\infty$-shift (adapted to some set $\left\{x_{k}^{\ell}\right\}$ ) and if $\lambda \in \mathbb{C}$ with $|\lambda|=1$, then $\lambda+S \in \mathcal{M i x}(X)$.

Proof. This follows from Theorem 2.3 and Lemma 2.2

\section{Approximating operators With Dense Generalised KERNEL}

Our aim now is to show that if an operator $S \in \mathcal{B}(X)$ (with $X$ as usual a separable infinite dimensional Banach space) has dense generalised kernel then it can be approximated in norm by generalised shifts (as defined in Definition 2.1). Our approach is to first find linearly independent vectors

$$
\left\{y_{j}^{\ell}: 1 \leq \ell<\infty, 1 \leq j \leq d_{\ell}\right\}
$$

with dense linear span in $X$ which are well adapted to $S$ in a sense somewhat similar to a forward shift version of (2.1). There will however 
be some important differences between what we can achieve for general $S$ and what is required in (2.1). In particular, the vectors $y_{j}^{\ell}$ will not necessarily belong to $\mathcal{R}^{\infty}(S)$ and our approach will be to adjust the operator $S$ (with an adjustment of at most a prescribed $\varepsilon>0$ in norm) so as to be able to apply Corollary 2.4.

For any sequence or set of vectors $\left\{x_{n}\right\}$ we will use square brackets $\left[x_{n}\right]$ for the closure of their linear span.

We describe our construction as an algorithm, starting from a fixed sequence $\left\{x_{n}: n \in \mathbb{N}\right\} \subset \mathcal{N}^{\infty}(S)$ so that $\left[x_{n}: n \in \mathbb{N}\right]=X$ (which certainly exists since $X$ is assumed to be separable). We suppose also that $\left\{x_{n}: n \in \mathbb{N}\right\}$ is algebraicly linearly independent (or at least that $\left.x_{1} \neq 0\right)$.

We begin by setting

$$
y_{1}^{1}=x_{1} .
$$

Then there is a smallest $r \geq 1$ with

$$
S^{r} y_{1}^{1}=0 .
$$

and we choose $d_{1}=r$. Then the space $E_{1}=\operatorname{span}\left\{x_{1}, S x_{1}, \ldots, S^{r-1} x_{1}\right\}$ is $S$-invariant and we let

$$
y_{j}^{1}=S^{j-1} y_{1}^{1} \quad\left(2 \leq j \leq d_{1}\right) .
$$

Assuming that we have already chosen $d_{1}, \ldots, d_{\ell} \in \mathbb{N}$ and vectors $\left\{y_{j}^{i}: 1 \leq i \leq \ell, 1 \leq j<d_{i}+1\right\}$, we will use the notation

$$
E_{m}=\left[y_{j}^{i}: 1 \leq i \leq m, 1 \leq j \leq d_{i}\right]
$$

(even for $m=0$ when $E_{m}=\{0\}$ ) and insist during the algorithm that $S\left(E_{m}\right) \subseteq E_{m}$ holds for $m=1,2, \ldots, \ell$ as well as

$$
\begin{gathered}
S y_{j}^{m}=y_{j+1}^{m} \quad\left(1 \leq m \leq \ell, 1 \leq j<d_{m}\right), \\
S y_{d_{m}}^{m} \in E_{m-1} \quad(1 \leq m \leq \ell),
\end{gathered}
$$

and

$$
\operatorname{span}\left\{y_{j}^{m}: 1 \leq j \leq d_{m}\right\} \cap E_{m-1}=\{0\} \quad(1 \leq m \leq \ell) .
$$

We will have in addition that

$$
\begin{array}{cl}
\left\{x_{1}, \ldots, x_{m}\right\} \subset E_{m} & (1 \leq m \leq \ell) \\
y_{1}^{m} \in\left\{x_{1}, x_{2}, \ldots\right\} & (1 \leq m \leq \ell)
\end{array}
$$

and

$$
\left\{y_{j}^{m}: 1 \leq i \leq \ell, 1 \leq j \leq d_{m}\right\} \text { linearly independent. }
$$

Notice that the construction so far has ensured that these conditions hold initially for $\ell=1$. 
The next step is to choose the smallest $n$ such that $x_{n} \notin E_{\ell}$ and to put $y_{1}^{\ell+1}=x_{n}$ (which will ensure that (3.7) and (3.8 hold for $m=\ell+1$ ) and then there is a smallest $r \geq 1$ such that

$$
S^{r+1} y_{1}^{\ell+1} \in \operatorname{span}\left\{y_{1}^{\ell+1}, S y_{1}^{\ell+1}, S, \ldots, S^{r} y_{1}^{\ell+1}\right\}+E_{\ell} \quad(\forall r \in \mathbb{N})
$$

We put $d_{\ell+1}=r$. Then we take

$$
y_{j+1}^{\ell+1}=S^{j} y_{1}^{\ell+1} \quad\left(1 \leq j<d_{\ell+1}\right) .
$$

It is clear then that (3.4) and (3.6) hold for $m=\ell+1$. To show (3.5), we can consider the operator induced by $S$ on the quotient $X / E_{\ell}$, which we temporarily denote $\tilde{S}$. Writing $\tilde{x}$ for the coset $x+E_{\ell}$ we then have $\tilde{S}(\tilde{x})=\widetilde{S x}$ and $\tilde{S}$ is a nilpotent operator with an invariant subspace $\operatorname{span}\left\{\widetilde{y_{j}^{l+1}}: 1 \leq j \leq r\right\}$ of dimension $r$. Hence $\tilde{S}^{r}=0$ on this subspace and (3.5) holds for $m=\ell+1$, so that also $T\left(E_{\ell+1}\right) \subseteq E_{\ell+1}$. Finally, (3.9) is easy to check for $m=\ell+1$.

Theorem 3.1. Let $X$ be a separable infinite dimensional Banach space and let $S \in \mathcal{B}(X)$ be such that $\mathcal{N}^{\infty}(S)$ is dense in $X$. Then $S$ is in the norm closure of the generalised backward 1-shift operators on $X$ so $T=\lambda+S \in \overline{\mathcal{M i x}(X)}$ (the norm closure of $\mathcal{M i x}(X)$ ) for each $\lambda \in \mathbb{C}$ with $|\lambda|=1$.

Proof. Let $\left\{x_{n}\right\}_{n=1}^{\infty} \subset \mathcal{N}^{\infty}(T)$ be a linearly independent set whose span is dense in $X$. We now apply the above construction to produce vectors $\left\{y_{j}^{\ell}: 1 \leq \ell<L+1,1 \leq j \leq d_{\ell}\right\}$ which satisfy the conditions (3.4) through (3.9) for all $\ell<\infty$.

For each $(\ell, j)$ with $1 \leq j \leq d_{\ell}$, we can use the Hahn-Banach theorem and choose $y_{j}^{\ell *} \in X^{*}$ (the dual space of $X$ ) so that

$$
y_{j}^{\ell *}\left(y_{k}^{m}\right)=\delta_{\ell, n} \delta_{j, k} \text { if } m \leq \ell .
$$

Given any $\varepsilon>0$, we then choose $\varepsilon_{\ell}>0$ such that

$$
\sum_{\ell=1}^{\infty} \varepsilon_{\ell}\left\|y_{1}^{\ell+1 *}\right\|\left\|y_{d_{\ell}}^{\ell}\right\|<\varepsilon .
$$

We now define $S_{\ell}: X \rightarrow X$ for $\ell \geq 2$ by $S_{\ell}=0$ if $y_{1}^{\ell-1, *}\left(S y_{d_{\ell}}^{\ell}\right) \neq 0$ and $S_{\ell}(x)=\varepsilon_{\ell} y_{1}^{\ell-1, *}(x) y_{d_{\ell}}^{\ell}$ otherwise. Then $\left\|S_{\ell}\right\| \leq \varepsilon_{\ell}\left\|y_{1}^{\ell-1 *}\right\|\left\|y_{d_{\ell}}^{\ell}\right\|$ and so $\sum_{\ell=1}^{\infty}\left\|S_{\ell}\right\|<\varepsilon$. Put $S^{\prime}=S+\sum_{\ell=2}^{\infty} S_{\ell}$.

We claim that $S^{\prime}$ is then a generalised 1-shift in the sense of Definition 2.1 with respect to the set obtained by linearly ordering $\left\{y_{j}^{\ell}: \ell \in\right.$ $\left.\mathbb{N}, 1 \leq j \leq d_{\ell}\right\}$ by taking those vectors in the order

$$
y_{d_{1}}^{1}, \ldots, y_{1}^{1}, y_{d_{2}}^{2}, \ldots, y_{1}^{2}, y_{d_{3}}^{3}, \ldots
$$

To verify this notice that for $(\ell, 1) \leq(k, j)$ we have $S_{\ell}\left(y_{j}^{k}\right) \in E_{\ell-1}$ but $S_{\ell}\left(y_{j}^{k}\right)=0$ if $\ell>k$. Hence $S^{\prime}\left(y_{j}^{k}\right)-y_{j+1}^{k} \in E_{k-1}$ if $j<d_{k}$ by (3.4) while $S^{\prime}\left(y_{j}^{k}\right) \in E_{k-1}$ if $j=d_{k}$ by (3.5). We have organised that 
$y_{1}^{\ell-1, *}\left(S^{\prime} y_{d_{\ell}}^{\ell}\right) \neq 0$ for $\ell \geq 2$, which is the remaining condition needed for $S^{\prime}$ to be a generalised 1-shift.

Since, for $|\lambda|=1$, we have $\lambda+S^{\prime} \in \mathcal{M i x}(X)$ by Corollary 2.4, and $\left\|(\lambda+S)-\left(\lambda+S^{\prime}\right)\right\|<\varepsilon$, we conclude that $\lambda+S^{\prime} \in \overline{\mathcal{M i x}(X)}$ as required.

Corollary 3.2. Let $X$ be a separable infinite dimensional Banach space and let $N \in \mathcal{B}(X)$ be a nilpotent. Then $\lambda+N \in \overline{\mathcal{M i x}(X)}$.

We say that a sequence of vectors $\left\{x_{n}\right\}_{n=1}^{\infty}$ is minimal if there exist functionals $x_{n}^{*} \in X^{*}$ such that $x_{n}^{*}\left(x_{m}\right)=\delta_{n, m}$ and a minimal sequence of vectors is fundamental if its linear span is dense in $X$. The $x_{n}^{*}$ are known as biorthogonal functionals for $\left\{x_{n}\right\}_{n=1}^{\infty}$. We call $\left\{x_{n}\right\}_{n=1}^{\infty}$ normalised if $\left\|x_{n}\right\|=1$ for each $n$. In particular a Schauder basis is a minimal fundamental sequence.

By forward shift with respect to a basis $\left\{x_{n}\right\}_{n=1}^{\infty}$ we mean an operator $S \in \mathcal{B}(X)$ satisfying $S x_{n}=w_{n} x_{n+1}$ for $n \geq 1$, where $w_{n} \in \mathbb{C}$ are weights. We will also consider bilateral shifts when the basis is $\left\{x_{n}\right\}_{n=-\infty}^{\infty}$.

In the next Proposition we consider a more general type of operator akin to a mixture of shifts but we do not insist on a Schauder basis.

Proposition 3.3. Let $X$ be a separable infinite dimensional Banach space. Suppose $S \in B(X)$ is an operator such that

$$
S x_{j}^{i}=a_{j}^{i} x_{j+1}^{i} \text { and } S y_{j}^{i}=b_{j}^{i} y_{j+1}^{i}
$$

where $a_{j}^{i}, b_{j}^{i} \in \mathbb{K}$ and

$\left\{x_{j}^{i}: 1 \leq i<I_{1}+1,1 \leq j<\infty\right\} \cup\left\{y_{j}^{i}: 1 \leq i<I_{2}+1,-\infty<j<\infty\right\}$ is a normalised minimal fundamental set for $X$ with biorthogonal functionals

$\left\{x_{j}^{i *}: 1 \leq i<I_{1}+1,1 \leq j<\infty\right\} \cup\left\{y_{j}^{i *}: 1 \leq i<I_{2}+1,-\infty<j<\infty\right\}$ where $0 \leq I_{1}, I_{2} \leq \infty$ (but we can't have $I_{1}=I_{2}=0$ ).

If for each $i$ with $1 \leq i<I_{1}+1$ there is an increasing sequence $n_{j}^{i}$ with $\left|a_{n_{j}^{i}}^{i}\right|\left\|x_{n_{j}^{i}}^{i}\right\| \rightarrow 0$ and for each $i$ with $1 \leq i<I_{2}+1$ there is an increasing sequence $m_{j}^{i}$ with $\left|b_{m_{j}^{i}}^{i}\right|\left\|y_{m_{j}^{i}}^{i}\right\| \rightarrow 0$ then $\lambda+S$ is in the closure of the mixing operators.

Proof. Let $\epsilon>0$ be given and let $\epsilon_{j}^{i}>0, \delta_{j}^{i}>0$ be such that

$$
\sum_{i=1}^{I_{1}} \sum_{j=1}^{\infty} \epsilon_{j}^{i}+\sum_{i=1}^{I_{2}} \sum_{j=1}^{\infty} \delta_{j}^{i}<\epsilon / 2 .
$$

We have that $\left|a_{n_{j}^{i}}^{i}\right|\left\|x_{n_{j}^{i}}^{i *}\right\| \rightarrow 0$ so for each $i$ choose a subsequence $n_{k_{j}}^{i}$ such that $\left|a_{n_{k_{j}}^{i}}^{i}\right|\left\|x_{n_{k_{j}}^{i}}^{i *}\right\|<\epsilon_{j}^{i}$ and similarly choose a subsequence $m_{k_{j}}^{i}$ 
such that $\left|b_{m_{k_{j}}^{i}}^{i}\right|\left\|y_{m_{k_{j}}^{i}}^{i *}\right\|<\delta_{j}^{i}$. Let

$$
K=\sum_{i=1}^{I_{1}} \sum_{j=1}^{\infty} a_{n_{k_{j}}^{i}}^{i} x_{n_{k_{j}}^{i}}^{i *} \otimes x_{n_{k_{j}}^{i}+1}^{i}+\sum_{i=1}^{I_{2}} \sum_{j=1}^{\infty} b_{m_{k_{j}}^{i}}^{i} y_{m_{k_{j}}^{i}}^{i *} \otimes y_{m_{k_{j}}^{i}+1}^{i} .
$$

Then

$$
\begin{aligned}
\|K\| & \leq \sum_{i=1}^{I_{1}} \sum_{j=1}^{\infty}\left|a_{n_{k_{j}}^{i}}^{i}\right|\left\|x_{n_{k_{j}}^{i}}^{i *}\right\|+\sum_{i=1}^{I_{2}} \sum_{j=1}^{\infty}\left|b_{m_{k_{j}}^{i}}^{i}\right|\left\|y_{m_{k_{j}}^{i}}^{i *}\right\| \\
& <\sum_{i=1}^{I_{1}} \sum_{j=1}^{\infty} \epsilon_{j}^{i}+\sum_{i=1}^{I_{2}} \sum_{j=1}^{\infty} \delta_{j}^{i}<\epsilon / 2
\end{aligned}
$$

Claim. $\mathcal{N}^{\infty}(S-K)$ is dense in $X$.

Proof. First, we will show that $x_{j}^{i} \in \mathcal{N}^{\infty}(S-K)$ for each $i, j$. Note that $(S-K) x_{j}^{i}=S x_{j}^{i}=a_{j}^{i} x_{j+1}^{i}$ for $(i, j) \neq\left(i, n_{k_{m}}^{i}\right)$. We have $(S-K) x_{n_{k_{j}}^{i}}^{i}=a_{n_{k_{j}}^{i}}^{i} x_{n_{k_{j}}^{i}+1}^{i}-$ $a_{n_{k_{j}}}^{i} x_{n_{k_{j}}+1}^{i}=0$. Suppose $n_{k_{j}}^{i}<j \leq m_{k}^{i}$, say $j=m_{k}^{i}-\ell$, then $\left.(S-K)^{\ell+1} x_{j}^{i}=\left(a_{j}^{i} a_{j+1}^{i} \ldots a_{m_{k}^{i}-1}^{i}\right)(S-K) x_{j+l}^{i}\right)=0$. So $x_{j}^{i} \in \mathcal{N}^{\infty}(S-K)$.

Similarly $y_{j}^{i} \in \mathcal{N}^{\infty}(S-K)$ for $j \geq m_{1}^{i}$. But we also have that $(S-K) y_{j}^{i}=S y_{j}^{i}$ for $j<m_{1}^{i}$. Let $j=m_{1}^{i}-\ell$ for some $\ell \in \mathbb{N}$. Then $(S-K)^{\ell+1} y_{j}^{i}=\left(b_{j}^{i} b_{j+1}^{i} \ldots b_{m_{1}^{i}-1}^{i}\right)(S-$ $K) y_{j+l}^{i}=0$ so $y_{j}^{i} \in \mathcal{N}^{\infty}(S-K)$.

Then $\operatorname{span}\left\{x_{j}^{i}\right\} \cup\left\{y_{j}^{i}\right\} \subset \mathcal{N}^{\infty}(S-K)$ and therefore $\mathcal{N}^{\infty}(S-K)$ is dense in $X$.

Therefore by Theorem 3.1 we have that there is a 1-shift $B$ such that $\|S-K-B\|<\epsilon / 2$. So $\|S-B\| \leq\|S-(S-K)\|+\|(S-K)-B\|<\epsilon$ and therefore (via Corollary 2.4) $\lambda+S$ is in the closure of the mixing operators.

Corollary 3.4. Let $X$ be a separable infinite dimensional Banach space with a Schauder basis. If $S$ is a weighted forward or bilateral shift on a normalised basis for $X$ with weights $w_{j}$ such that there exists an increasing sequence $n_{j}$ with $\left|w_{n_{j}}\right| \rightarrow 0$ then $\lambda+S$ is in the closure of the mixing operators.

In particular if $S$ is a quasinilpotent forward or bilateral shift on a normalised basis then $\lambda+S$ is in the closure of the mixing operators.

Proof. Suppose $e_{n}$ is our normalised basis. By a remark at the beginning of [7, 1.b] we have that $\sup _{n}\left\|e_{n}^{*}\right\|=K<\infty$. Then $\left|w_{n_{j}}\right|\left\|e_{n}^{*}\right\| \leq$ $K\left|w_{n_{j}}\right| \rightarrow 0$ and the rest follows from Proposition 3.3 by setting $I_{1}=1$, 
$I_{2}=0$ and $x_{j}^{1}=e_{j}$ for the forward shift case and $I_{1}=0, I_{2}=1$ and $y_{j}^{1}=e_{j}$ for the bilateral shift case.

For the second part we need only show that if the shift is quasinilpotent then there must be a subsequence of the weights going to 0. Assume for the sake of contradiction that this is not true. Then there is $j \in \mathbb{N}$ such that $C=\inf _{n \geq j}\left\{\left|w_{n}\right|\right\} \neq 0$. Choose $0<\epsilon<C$. Then there is $N \in \mathbb{N}$ such that $\left\|S^{n} x\right\|<\epsilon^{n}\|x\|$ for all $n>N$.

$$
C^{n} \leq\left\|w_{j} w_{j+1} \ldots w_{j+n-1} e_{j+n}\right\|=\left\|S^{n} e_{j}\right\|<\epsilon^{n}<C^{n}
$$

for $n>N$.

\section{REFERENCES}

[1] C. Apostol, L. A. Fialkow, D. A. Herrero and D. Voiculescu, Approximation of Hilbert space operators. Vol. II, Research Notes in Mathematics, vol. 102, Pitman (Advanced Publishing Program), Boston, MA (1984).

[2] S. A. Argyros and R. G. Haydon, A hereditarily indecomposable $\mathscr{L}_{\infty}$-space that solves the scalar-plus-compact problem, Acta Math. 206 (2011) 1-54, URL http://dx.doi.org/10.1007/s11511-011-0058-y.

[3] F. Bayart and É. Matheron, Dynamics of linear operators, Cambridge Tracts in Mathematics, vol. 179, Cambridge University Press, Cambridge (2009), URL http://dx.doi.org/10.1017/CB09780511581113.

[4] D. W. Hadwin, E. A. Nordgren, H. Radjavi and P. Rosenthal, Most similarity orbits are strongly dense, Proc. Amer. Math. Soc. 76 (1979) 250-252, URL http://dx.doi.org/10.2307/2042997.

[5] D. A. Herrero, Approximation of Hilbert space operators. Vol. 1, Pitman Research Notes in Mathematics Series, vol. 224, Longman Scientific \& Technical, Harlow; copublished in the United States with John Wiley \& Sons, Inc., New York, second ed. (1989).

[6] -, Limits of hypercyclic and supercyclic operators, J. Funct. Anal. 99 (1991) 179-190, URL http://dx.doi.org/10.1016/0022-1236(91)90058-D

[7] J. Lindenstrauss and L. Tzafriri, Clasical Banach Spaces I, Ergebnisse der Mathematik und ihrer Grenzgebiete, Vol. 92. Springer-Verlag, Berlin-New York, 1977.

[8] V. Müller, On the Salas theorem and hypercyclicity of $f(T)$, Integral Equations Operator Theory $\mathbf{6 7}$ (2010) 439-448, URL http://dx.doi.org/10.1007/s00020-010-1791-x.

School of Mathematics, Trinity College, Dublin 2

E-mail address: jaboland@tcd.ie 